\title{
Photonic Crystal-Coupler for Sheet Beam THz Vacuum Electron Tubes
}

\author{
Rosa Letizia, Member, IEEE, Mauro Mineo and Claudio Paoloni, Senior Member, IEEE
}

\begin{abstract}
Photonic Crystal (PhC) technology was recently proposed as a compact and effective solution to improve the performance and ease the fabrication of terahertz vacuum electron devices. In particular, the introduction of defects in a bidimensional, all metallic $\mathrm{PhC}$ provides very effective transmission of the useful signal in a compact input/output coupler where a novel tunnel for sheet-beam injection/collection is realized. Simulation results are here experimentally validated via measurements on a Ku-band scaled model of the PhC-coupler which confirm the validity of the concept.
\end{abstract}

Index Terms-Photonic Crystals (PhCs), Terahertz, vacuum electron devices, couplers, sheet beam.

\section{INTRODUCTION}

A S novel technological solutions to design portable sub$\mathrm{THz}$ and $\mathrm{THz}(100-1000 \mathrm{GHz})$ vacuum electron devices with relatively high output power are sought, photonic bandgap (PBG) structures have recently attracted the interest of the vacuum electron device (VED) community, in the emerging field of millimeter wave sources and amplifiers [1][5]. At THz frequency, many challenges need to be overcome to offer viable solutions: assembly of the slow wave structure (SWS) with dimensions of parts in the order of tens of microns, where a suitable level of vacuum is obtained, is particularly critical, while the achievable output power is limited by the reduced beam transversal section. Moreover, the performance of THz VEDs is affected by the reduced intrinsic conductivity of the metal and by the ohmic losses deriving from the metal wall surface roughness.

In our early work, we proposed the use of photonic crystal (PhC) technology to assist the design of THz VEDs based on SWSs as traveling wave tubes (TWTs) and backward wave oscillators (BWOs), [4]. PhCs are a well-known technology for the engineering of frequency-selective synthetic media by means of the property of exhibiting a photonic bandgap [6][8]. The use of $\mathrm{PhC}$ technology is proposed to relax some of the issues which make the realization of conventional SWSs and coupler for $\mathrm{THz}$ vacuum electron devices very demanding. In particular, the properties of PhCs solve for the

Manuscript received

This work was supported by EPSRC grant EP/L026597/1.

R. Letizia and C. Paoloni are with the Engineering Department, Lancaster University, Lancaster, LA1 4YW, UK (e-mail: r.letizia@lancaster.ac.uk).

M. Mineo is with e2v Technologies, Chelmsford.

R. Letizia is also with The Cockcroft Institute, Daresbury, WA4 4AD, UK. problem of beam tunnel cut-off for large sheet electron beams (up to 15:1 ratio) which have been demonstrated to enhance VEDs performance [6]-[13]. In this context, SWSs are typically derived from the corrugated waveguide (CRW). $\mathrm{PhCs}$ have been experimentally demonstrated in the X-band as a solution to eliminate currents in joints or interfaces of enclosed metallic waveguides [14], simplifying significantly the assembly. In addition, thanks to their open nature, PhCwaveguides introduce a wider empty space around the interaction region which can ease the process of vacuum pumping.

In this paper, the experimental validation of the novel coupler based on a 2D PhC-bend scaled for the Ku-band is discussed. Measurement results demonstrate that the concept is valid and may be successfully scaled to the $\mathrm{THz}$ frequency range. The low sensitivity due to a gap between PhC pillars and top lid which could further ease the assembly is also demonstrated in simulation.

\section{PHC COUPLER FOR SHEET BEAM THZ SWSS}

A typical configuration of the corrugated waveguide and coupler to support a sheet electron beam and extract/inject RF signal from/to an input/output port is shown in Fig.1. The wide cross section of the beam tunnel required in sheet beam VEDs, behaves as a waveguide over cutoff, leading to a relevant portion of the signal propagating in the beam tunnel rather than in the coupler port, with consequent degradation of performance (Fig.1). So far, appropriate impedance mismatches have been added in the beam tunnel to obtain the

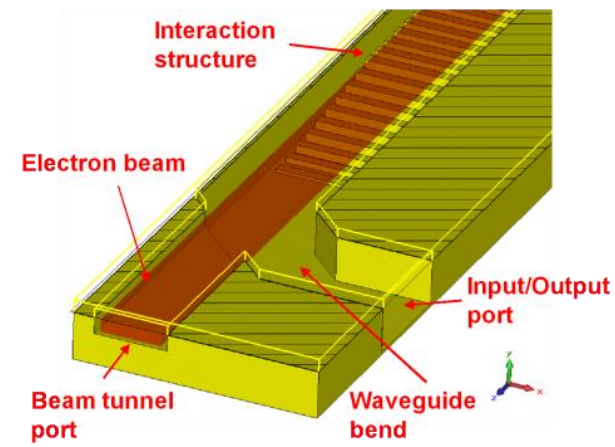

Fig. 1. Schematic diagram of a typical coupler for a sheet beam in a CRW.

required isolation of the beam aperture [15]. However, this approach results in an increased complexity of geometries and fabrication. The Photonic bandgap has been demonstrated as an effective solution to build high quality couplers with wide 
beam tunnel of ratio up to 15:1 [4]. The SWS taken into consideration is a CRW as shown in Fig. 1, whose typical dispersion curve is shown in Fig. 2(a). The principle is to replace the metal walls with a $\mathrm{PhC}$ designed around the operation frequency to realize the effective and selective confinement of the hybrid modes supported by the CRW [4]. Depending on the region of beam intersection chosen, the SWS can realize a backward wave oscillator (BWO) or a travelling wave tube (TWT) for which the design here presented can operate as input or output coupler.

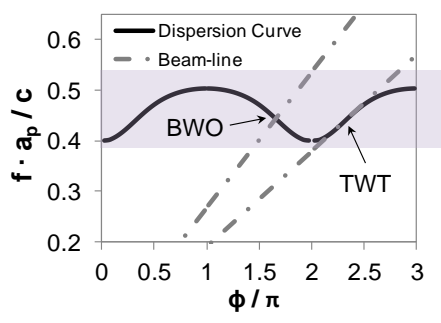

(a)

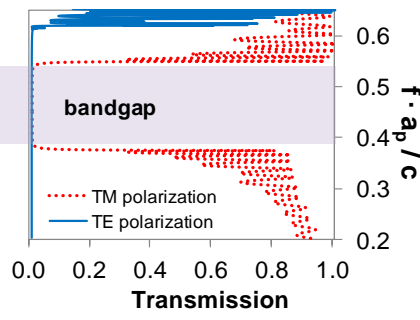

(b)
Fig. 2. (a): typical dispersion curve of a CRW with beam line at two different voltages to highlight BWO and TWT regimes. (b): transmission coefficient of the designed $\mathrm{PhC}$.

The $\mathrm{PhC}$ is made of a square lattice of squared (or circular) copper pillars designed to exhibit a photonic bandgap for both $\mathrm{TE}$ and TM polarization of the incident electromagnetic field between 0.4 and 0.54 in terms of normalized frequency $(f$. $a_{p} / c$ ), where $a_{p}$ represents the period of the $\mathrm{PhC}$ and the pillars edge length is taken as $s_{p}=0.42 a_{p}$, (Fig. 2(b)). The bandgap can be tuned to the frequency range $0.55-0.8 \mathrm{THz}$ when the period, $a_{p}$, is chosen as $204 \mu \mathrm{m}$. Fig. 3 shows the proposed design for the three-port $\mathrm{PhC}$-coupler, based on a $90^{\circ}$ bend of the single line defect waveguide [6]-[7]. Point defects (pillars with different dimension and position) are introduced around the bend in order to maximize transmission from Port 1 (SWS) to Port 2 (input/output port) and vice versa, for a given width of the $\mathrm{PhC}$ waveguide. Four properly modified pillars are introduced in the beam tunnel (towards Port 3) to allow the beam through while retaining the photonic bandgap around operation. The inner corner pillar (Fig. 3, $\mathrm{P}_{1}$ ), is offset of $0.20 a_{p}$, and the position of the pillars $\mathrm{P}_{2}$ and $\mathrm{P}_{4-9}$ is offset of $0.33 a_{p}$ with respect to the $\mathrm{PhC}$ lattice. In addition, the outer corner pillar and the beam tunnel corrugations (Fig. 3, $\mathrm{P}_{2}, \mathrm{P}_{6-9}$ ) have $0.43 a_{p}$ height, $0.50 a_{p}$ length and $0.42 a_{p}$ width. The dimensions are fully compatible with microfabrication processes, such as UV-LIGA or CNC milling, depending on the operating frequency, [16]. The modified $\mathrm{PhC}$ pillars with different height in the outer corner and beam tunnel have the same height of the corrugated waveguide teeth which provides synchronism at the operation frequency. To realize the structure via LIGA process, a two-step approach can be used for the $\mathrm{PhC}$ pillars and the defects/central corrugations. Simulation results in [4] showed that the PhC-bend has a symmetrical behavior and can be used as a coupler for forward (Port 2 towards Port 1) and backward operation (Port 1 towards Port 2). Also, transmission from Port 3 towards Port 2 and 1 was found to be below $-45 \mathrm{~dB}$ at $0.65 \mathrm{THz}$ which predicts with high degree of accuracy high isolation of Port 3.

\section{FABRICATION AND EXPERIMENTAL MEASUREMENTS OF THE KU-BAND SCALED MODEL}

In order to validate the results obtained from numerical simulations, the PhC-coupler design at $0.65 \mathrm{THz}$ from [4] has been scaled down and fabricated in Aluminum to operate in the Ku-band, with a central operating frequency of $14 \mathrm{GHz}$. Due to the nature of PhC-technology, the scaling is a reliable procedure for the demonstration of the structure properties. To tune the design, a period $a_{p}=9.47 \mathrm{~mm}$ is chosen, feasible by a common CNC milling machine. The width of the linear defect forming the waveguide is given as $15.8 \mathrm{~mm}$ in order to accommodate for the WR62 flanges (Fig. 4).

Figure 5(a)-(c) show the measured $S$-parameters for the $\mathrm{Ku}$ band PhC-coupler when excitation is sent from Port 1, Port 2, and Port 3, respectively. As it can be noted, the behavior from

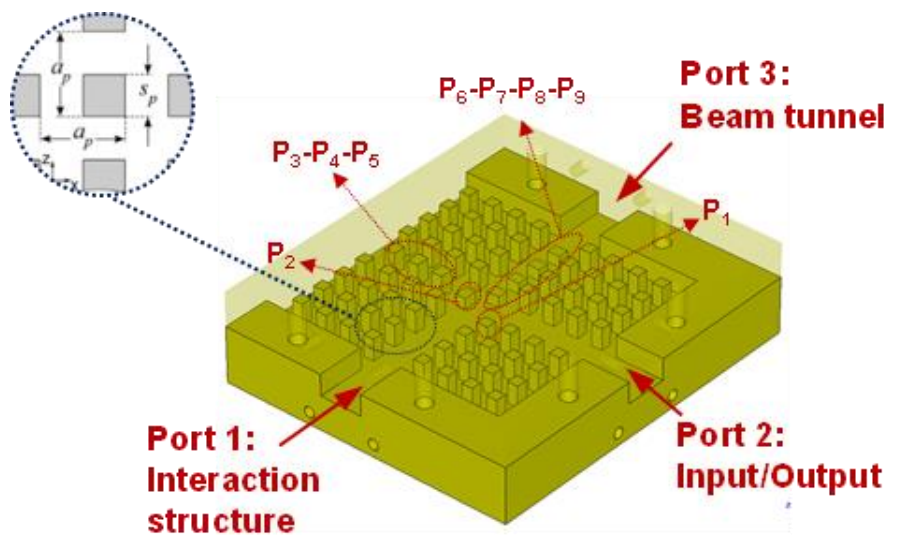

Fig. 3. Perspective view of the input/output PhC-coupler.

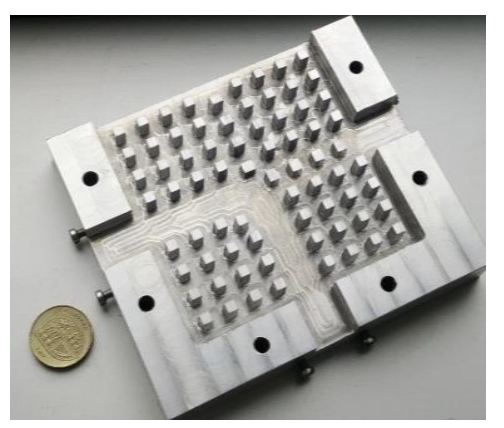

Fig. 4. Ku-band model of the PhC-coupler fabricated in $\mathrm{Al}$ with the $\mathrm{PhC}$ period is $a_{p}=9.47 \mathrm{~mm}$.

Port 1 and 2 is symmetrical, with a value of return loss of -20 $\mathrm{dB}$ in a wide frequency band centered at $14 \mathrm{GHz}$. This results in a wide tunability range of the operating frequency of the whole VED. Three-dimensional electromagnetic simulations performed by CST Microwave Studio [17] are compared with the experimental results shown in Fig. 5(d)-(f), respectively. The very good agreement of measured $S$-parameters with numerical simulations demonstrates the validity of the novel coupler approach based on $\mathrm{PhC}$ technology and the simulator reliability. In particular, both measured transmission parameters, $\left|S_{12}\right|$ and $\left|S_{21}\right|$, are very close to the values predicted in simulation with an average difference less than $0.9 \mathrm{~dB}$ across the band. Measurements of $\left|S_{33}\right|$ for the isolated port match even more closely the simulation results with an 

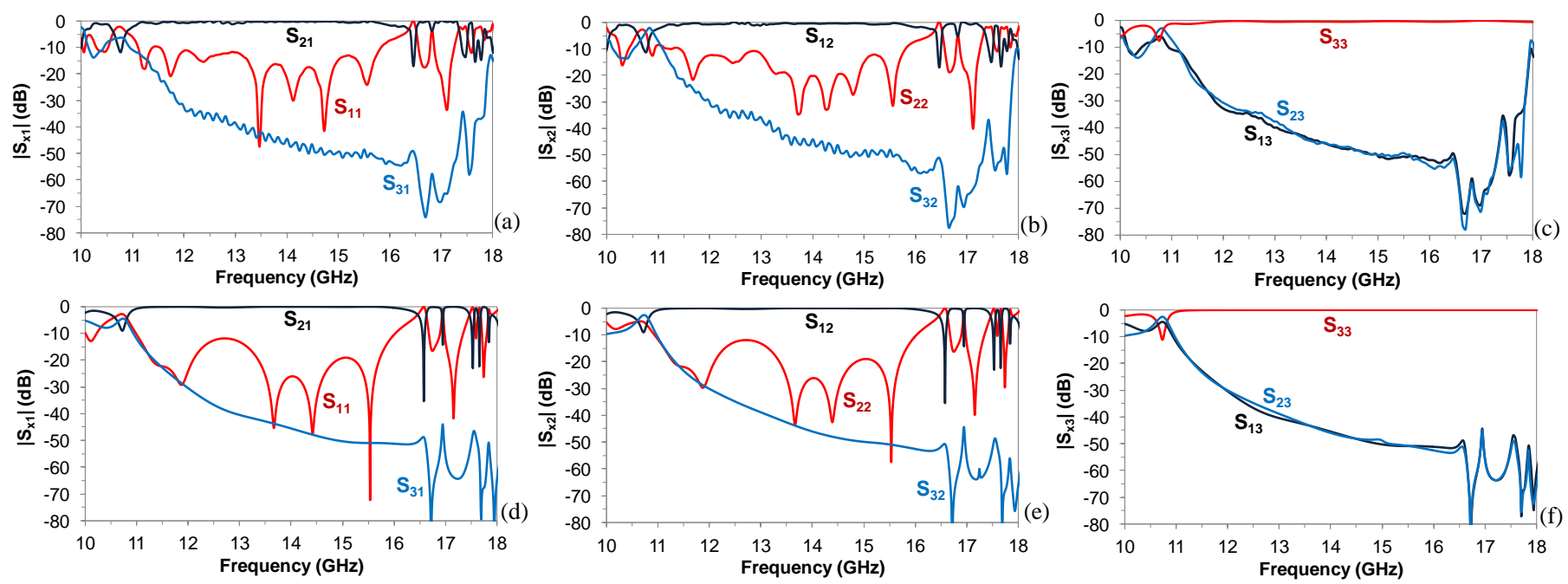

Fig. 5. (a), (b) and (c): measured S-parameters; (d), (e) and (f): simulated S-parameters of the Ku-band PhC coupler.

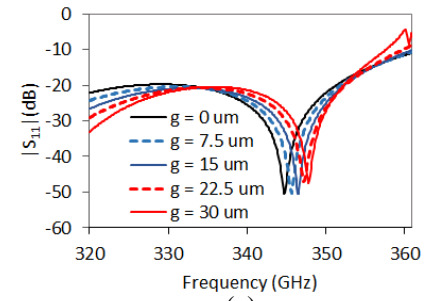

(a)

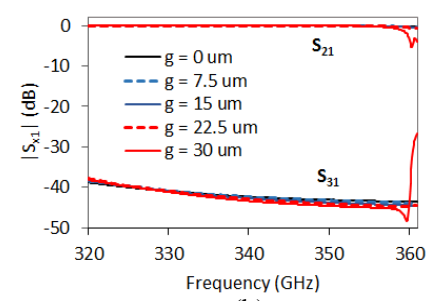

(b)
Fig. 6. (a): $S_{11}$ and (b): $S_{21}-S_{31}$ parameters for the PhC-coupler centered at 346 $\mathrm{GHz}$ with a gap between the pillars and the top lid varying between 0-30 $\mu \mathrm{m}$

agreement better than $0.5 \mathrm{~dB}$.

\section{SENSITIVITY TO GAP BETWEEN TOP LID AND PHC PILLARS}

The PhC-coupler was shown to have very low sensitivity against tolerances on the defect geometry, [4]. Here, the robustness of the design when a gap between the $\mathrm{PhC}$ pillars and the top lid exists is investigated. The case of PhC-coupler tuned at $346 \mathrm{GHz}$ is chosen with $a_{p}=0.4 \mathrm{~mm}, s_{p}=0.167 \mathrm{~mm}$ and pillar height of $0.36 \mathrm{~mm}$. $S$-parameters are simulated for this structure when the gap, $g$, is varied in a range 0-30 $\mu \mathrm{m}$. The conductivity of copper is assumed as $\sigma_{C u}=3.907 \times 10^{7}$ $\mathrm{S} / \mathrm{m}$, lower than the ideal value, to account for metal losses in simulation. Figure 6(a)-(b) show that return loss for the coupler is maintained below $-20 \mathrm{~dB}$ between $320-355 \mathrm{GHz}$ and $\left|S_{31}\right|$ is as low as $-40 \mathrm{~dB}$, with no significant changes with respect to the case where no gap exists (black line in Fig. 6(a)(b)). The obtained results indicate that the performance of the coupler is robust in the range of operation with very low sensitivity to a gap between $\mathrm{PhC}$ pillars and top lid, making the assembly of the overall structure easier.

\section{CONCLUSIONS}

A novel approach to the design of a high performance input/output coupler for $\mathrm{THz}$ vacuum electron devices based on wide sheet beams has been presented. By taking advantage of the easy scalability of $\mathrm{PhCs}$ bandgap properties, the simulated results have been validated against measurement results for a prototype realized at $14 \mathrm{GHz}$. Low sensitivity to the existence of a gap between PhC pillars and top lid up to 30 $\mu m$ is numerically predicted at $346 \mathrm{GHz}$ indicating robustness of the design to fabrication tolerances and a possibility for easier assembly in $\mathrm{THz}$ VEDs.

\section{ACKNOWLEDGMENT}

The Authors would like to thank Jonathan Gates for the realization of the Ku-band prototype.

\section{REFERENCES}

[1] G. O. Vela, M. S. Miller, R. W. Grow, and J. M. Baird, "Terahertz backward-wave oscillators with photonic crystal waveguides," in IEEE Proc. of IVEC/IVESC, 2006, pp. 425-426, DOI: 10.1109/IVELEC.2006.1666364

[2] Y. Gong, H. Yin, Y. Wei, L. Yue, M. Deng, Z. Lu, X. Xu, W. Wang, P. Liu, and F. Liao, "Study of Traveling Wave Tube With FoldedWaveguide Circuit Shielded by Photonic Crystals," Electron Devices, IEEE Transactions on, vol.57, no.5, pp.1137-1145, May 2010, DOI: 10.1109/TED.2010.2043176

[3] Y.-M. Shin, "Frequency-selective plasmonic wave propagation through the overmoded waveguide with photonic-band-gap slab arrays," Phys. Plasmas, vol. 19 , no. 5, p. 053102,2012

[4] R. Letizia, M. Mineo, and C. Paoloni, "Photonic Crystal-Structures for THz Vacuum Electron Devices," Electron Devices, IEEE Transactions on, vol. 62, no.1, pp.178,183, Jan. 2015, DOI: 10.1109/TED.2014.2366639

[5] C. Paoloni, A. Di Carlo, F. Bouamrane, T. Bouvet, A. J. Durand, M. Kotiranta, V. Krozer, S. Megtert, M. Mineo, V. Zhurbenko, "Design and Realization Aspects of $1-\mathrm{THz}$ Cascade Backward Wave Amplifier Based on Double Corrugated Waveguide," Electron Devices, IEEE Transactions on, vol. 60, no. 3, pp. 1236-1243, Mar. 2013, DOI: 10.1109/TED.2013.2240686

[6] J. D. Joannopoulos, R. D. Meade, and J. N. Winn, Photonic Crystals: Molding the Flow of Light. Princeton, NJ, USA: Princeton Univ. Press, 1995

[7] S.-Y. Lin, E. Chow, V. Hietala, P. R. Villeneuve, and J. D. Joannopoulos, "Experimental Demonstration of Guiding and Bending of Electromagnetic Waves in a Photonic Crystal," Science, vol. 282, no. 5387, pp. 274-276, Oct. 1998, DOI: 10.1126/science.282.5387.274

[8] E. Degirmenci, F. Surre, S. Philippe, R. Maldonado-Basilio, and P. Landais., "Improved Bend Waveguide Design for Terahertz Transmission," IEEE Trans. Terahertz Sci. Technol., vol. 2, no. 1, pp. 137-143, Jan. 2012, DOI: 10.1109/TTHZ.2011.2177170

[9] K. T. Nguyen, J. A. Pasour, T. M. Antonsen, P. B. Larsen, J. J. Petillo, and B. Levush, "Intense Sheet Electron Beam Transport in a Uniform Solenoidal Magnetic Field," Electron Devices, IEEE Transactions on, 
vol. 56, no. 5, pp. 744-752, May 2009, DOI: 10.1109/TED.2009.2015420

[10] C. D. Joye, J. P. Calame, M. Garven and B. Levush "UV-LIGA microfabrication of $220 \mathrm{GHz}$ sheet beam amplifier gratings with SU-8 photoresist", J. Micromech. Microeng., vol. 20, no. 12, pp.125, 2010, DOI: $10.1109 /$ ICIMW.2010.5612561

[11] C. D. Joye, J. P. Calame, A. M. Cook, and M. Garven, "High-Power Copper Gratings for a Sheet-Beam Traveling-Wave Amplifier at Gband," Electron Devices, IEEE Transactions on , vol.60, no.1, pp.506509, Jan. 2013, DOI: 10.1109/TED.2012.2226591

[12] M. Mineo and C. Paoloni, "Corrugated rectangular waveguide tunable backward wave oscillator for THz applications", Electron Devices, IEEE Transactions on, vol. 57, pp. 1481-1484, 2010, DOI: 10.1109/TED.2010.2045678

[13] Z. Wang, Y. Gong, Y. Wei, Z. Duan, Y. Zhang, L. Yue, H. Gong, H. Yin, Z. Lu, J. Xu, and J. Feng, "High-Power Millimeter-Wave BWO Driven by Sheet Electron Beam," Electron Devices, IEEE Transactions on , vol.60, no.1, pp.471-477, Jan. 2013, DOI: 10.1109/TED.2012.2226587
[14] J. Hesler, "A photonic crystal joint (PCJ) for metal waveguides," Microwave symposium digest, 2001 IEEE MTT-S International, vol. 6., pp. 783-786, 2001, DOI: 10.1109/MWSYM.2001.967009

[15] Y.-M. Shin, A. Baig, R. Barchfeld, D. Gamzina, L. R. Barnett and N. C. Luhmann Jr., "Experimental study of multichromatic terahertz wave propagation through planar micro-channels," Appl. Phys. Lett., vol. 100, no. 15, pp. 154103, 2012, DOI: http://dx.doi.org/10.1063/1.3698362

[16] C. Paoloni, D. Gamzina, L. Himes, B. Popovic, R. Barchfeld, L. Yue, Y. Zheng, X. Tang, Y. Tang, P. Pan, H. Li, R. Letizia, M. Mineo, J. Feng, and N. C. Luhmann, Jr., "THz backward wave oscillators for plasma diagnostics in nuclear fusion", Plasma Science, IEEE Transactions on, vol. 44, no. 4, pp. 369-376, 2016, DOI: 10.1109/TPS.2016.2541119

[17] CST AG, Germany. CST STUDIO SUITE. [Online]. Available: http://www.cst.com 